\title{
Correction to: Estimating the Reproducibility of Experimental Philosophy
}

Florian Cova $^{1,2}\left(\mathbb{D} \cdot\right.$ Brent Strickland $^{3,4}$ - Angela Abatista ${ }^{5}$. Aurélien Allard ${ }^{6}$. James Andow ${ }^{7} \cdot$ Mario Attie $^{8}$ - James Beebe ${ }^{9} \cdot$ Renatas Berniūnas $^{10}$. Jordane Boudesseul $^{11}$ - Matteo Colombo ${ }^{12}$ - Fiery Cushman ${ }^{13} \cdot$ Rodrigo Diaz $^{14}$. Noah N'Djaye Nikolai van Dongen ${ }^{15}$ - Vilius Dranseika ${ }^{16}$ - Brian D. Earp ${ }^{17}$. Antonio Gaitán Torres ${ }^{18}$ - Ivar Hannikainen ${ }^{19}$ - José V. Hernández-Conde ${ }^{20}$. Wenjia $\mathrm{Hu}^{21}$ - François Jaquet ${ }^{1}$ - Kareem Khalifa ${ }^{22}$ - Hanna Kim ${ }^{23}$. Markus Kneer $^{24}$ • Joshua Knobe ${ }^{25}$ - Miklos Kurthy ${ }^{26}$ • Anthony Lantian ${ }^{27}$.

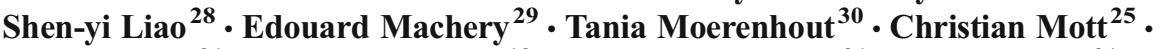
Mark Phelan ${ }^{21}$. Jonathan Phillips ${ }^{13}$ - Navin Rambharose ${ }^{21}$ - Kevin Reuter ${ }^{31}$. Felipe Romero $^{15}$ • Paulo Sousa ${ }^{32}$ • Jan Sprenger ${ }^{33}$ - Emile Thalabard ${ }^{34}$. Kevin Tobia $^{25} \cdot$ Hugo Viciana ${ }^{35} \cdot$ Daniel Wilkenfeld $^{29} \cdot$ Xiang Zhou $^{36}$

Correction to: Rev.Phil.Psych.

https://doi.org/10.1007/s13164-018-0400-9

Appendix 1 was incomplete in the initial online publication. The original article has been corrected.

The online version of the original article can be found at https://doi.org/10.1007/s13164-018-0400-9

Florian Cova

florian.cova@gmail.com

Brent Strickland

stricklandbrent@gmail.com

Angela Abatista

Angela.Abatista@unige.ch

Aurélien Allard

aurelien.ab.allard@gmail.com

James Andow

Jamesandow@gmail.com

Mario Attie

mario.attie@yale.edu 
James Beebe

jbeebe2@buffalo.edu

Renatas Berniūnas

renatasberniunas@gmail.com

Jordane Boudesseul

jmj.boudesseul@gmail.com

Matteo Colombo

M.Colombo@uvt.nl

Fiery Cushman

cushman@fas.harvard.edu

Rodrigo Diaz

rodrigo.diaz@philo.unibe.ch

Noah N'Djaye Nikolai van Dongen

n.djaye@gmail.com

Vilius Dranseika

vilius.dranseika@fsf.vu.lt

Brian D. Earp

brian.earp@gmail.com

Antonio Gaitán Torres

agaitan@hum.uc3m.es

Ivar Hannikainen

ivar.hannikainen@gmail.com

José V. Hernández-Conde

jhercon@gmail.com

Wenjia $\mathrm{Hu}$

wenjia.hu@lawrence.edu

François Jaquet

Francois.Jaquet@unige.ch

Kareem Khalifa

kkhalifa@middlebury.edu

Hanna Kim

hkim@washjeff.edu

Markus Kneer

markus.kneer@gmail.com

Joshua Knobe

joshua.knobe@yale.edu

Miklos Kurthy

mkurthy1@sheffield.ac.uk

Anthony Lantian

anthony.lantian@parisnanterre.fr

Shen-yi Liao

liao.shen.yi@gmail.com 
Edouard Machery
machery@pitt.edu
Tania Moerenhout
tania.moerenhout@ugent.be
Christian Mott
cjmott@gmail.com
Mark Phelan
phemark@gmail.com
Jonathan Phillips
phillips01@g.harvard.edu

Navin Rambharose

navin.rambharose@lawrence.edu

Kevin Reuter

kevin.reuter@philo.unibe.ch

Felipe Romero

F.Romero@uvt.nl

Paulo Sousa

paulo.sousa@qub.ac.uk

Jan Sprenger

jan.sprenger@unito.it

Emile Thalabard

emilio.thalabard@ laposte.net

Kevin Tobia

kevin.tobia@yale.edu

Hugo Viciana

Hviciana@iesa.csic.es

Daniel Wilkenfeld

dawilk@gmail.com

Xiang Zhou

xiangz@pitt.edu

1 Centre Interfacultaire en Sciences Affectives, Université de Genève, Geneva, Switzerland

2 Swiss Center for Affective Sciences, Campus Biotech, CISA - University of Geneva, Chemin des Mines, 9, 1202 Geneva, Switzerland

3 Département d'Etudes Cognitives, Ecole Normale Supérieure, Paris, France

4 Institut Jean Nicod, CNRS, Paris, France

5 Faculté de Psychologie et des Sciences de l'Education, Université de Genève, Geneva, Switzerland

6 Laboratoire des Théories du Politique, Université Paris 8 Vincennes, Saint-Denis, France

7 University of Reading, Reading, UK

8 Department of Philosophy, Yale University, New Haven, CT, USA

9 University at Buffalo, Buffalo, NY, USA 
10 Department of General Psychology, Vilnius University, Vilnius, Lithuania

11 Instituto de Investigación Científica, Universidad de Lima, Lima, Peru

12 Tilburg Center for Logic, Ethics and Philosophy of Science, Tilburg University, Tilburg, Netherlands

Department of Psychology, Harvard University, Cambridge, MA, USA

University of Bern, Bern, Switzerland

Tilburg University, Tilburg, Netherlands

Department of Logic and History of Philosophy, Faculty of Philosophy, Vilnius University, Vilnius, Lithuania Departments of Philosophy and Psychology, Yale University, New Haven, CT, USA

Departamento de Humanidades, Universidad Carlos III de Madrid, Getafe, Spain Catholic University of Rio de Janeiro, Rio de Janeiro, Brazil

Department of Linguistics and Basque Studies, University of the Basque Country, Leioa, Spain Lawrence University, Appleton, WI, USA

Philosophy Department, Middlebury College, Middlebury, VT, USA

Washington and Jefferson College, Washington, PA, USA

University of Zurich, Zurich, Switzerland

Yale University, New Haven, CT, USA

University of Sheffield, Sheffield, UK

Laboratoire Parisien de Psychologie Sociale, UPL, Université Paris Nanterre, Nanterre, France Department of Philosophy, University of Puget Sound, Tacoma, WA 98416, USA

Department of History and Philosophy of Science, Center for Philosophy of Science, University of Pittsburgh, Pittsburgh, PA, USA

Department of Philosophy and Moral Sciences and Department of Family Medicine and Primary Health Care, University of Ghent, Ghent, Belgium

31 Institute of Philosophy, University of Bern, Bern, Switzerland

32 Queen's University Belfast, Belfast, UK

33 Center for Logic, Language and Cognition, Department of Philosophy and Educational Sciences, University of Turin, Turin, Italy Juan de la Cierva Research Fellow, Instituto de Estudios Sociales Avanzados (IESA-CSIC), Córdoba, Spain 